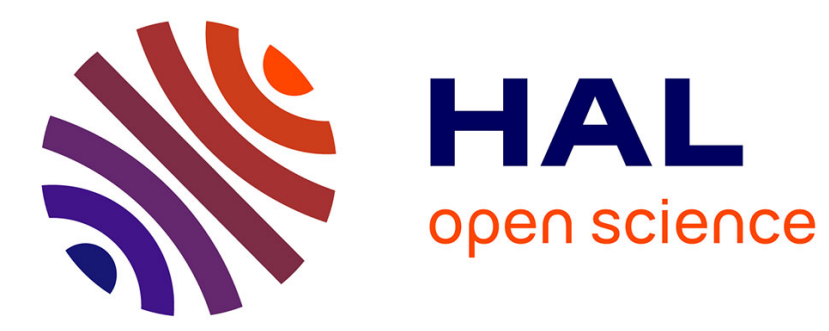

\title{
Oxydation du zircaloy-4 dans l'air à haute température
}

\author{
Sulistijono, A. Bouden, M. Cherfaoui, G. Beranger, M. Lambertin
}

\section{To cite this version:}

Sulistijono, A. Bouden, M. Cherfaoui, G. Beranger, M. Lambertin. Oxydation du zircaloy-4 dans l'air à haute température. Journal de Physique IV Proceedings, 1993, 03 (C9), pp.C9-439-C9-445. 10.1051/jp4:1993946 . jpa-00252386

\section{HAL Id: jpa-00252386 https://hal.science/jpa-00252386}

Submitted on 1 Jan 1993

HAL is a multi-disciplinary open access archive for the deposit and dissemination of scientific research documents, whether they are published or not. The documents may come from teaching and research institutions in France or abroad, or from public or private research centers.
L'archive ouverte pluridisciplinaire HAL, est destinée au dépôt et à la diffusion de documents scientifiques de niveau recherche, publiés ou non, émanant des établissements d'enseignement et de recherche français ou étrangers, des laboratoires publics ou privés. 


\title{
Oxydation du zircaloy-4 dans l'air à haute température
}

\author{
Sulistijono $\left({ }^{1}\right)$, A. Bouden $\left({ }^{1}\right)$, M. Cherfaoui $\left({ }^{2}\right)$, G. Beranger $\left({ }^{1}\right)$ et M. Lambertin $\left({ }^{1}\right)$
}

( ${ }^{1}$ LG2mS (URA 1505 CNRS), Université de Technologie de Compiègne, B.P. 649, F-60206 Compiègne Cedex, France

(2) CETIM, Service "Essais et Mesures Physiques", 52 Avenue Félix Louat, F-60304 Senlis, France

\begin{abstract}
The oxidation of zircaloy-4 in air was studied in the temperature range $350-750{ }^{\circ} \mathrm{C}$. The oxidation kinetics cannot be described by a single rate law. At the beginning of the reaction, the rate decreases regularly. Then, after a few hours, an acceleration is observed and finally a linear law is obeyed. The morphological observations show that the acceleration is related to the formation of cracks in the oxide scale. Moreover, two different zirconia phases (monoclinic and tetragonal) are identified. When the kinetic transition occurs, the tetragonal phase partially disappears. All these observations show that a mechanism, similar to that proposed to describe the oxidation of zircaloy-4 in steam, may be used to describe the reaction. In parallel, the oxidation reaction was studied by using acoustic emission. The frequency, the amplitude and the energy were used to characterize the signal. Typical groups were identified and their appearance was strongly correlated to the different parts of the reaction.
\end{abstract}

\section{Introduction.}

Lémission acoustique est une technique maintenant bien connue et bien maîtrisée pour détecter, dans le cas de structures métalliques, l'apparition et la propagation de défauts tels que les fissures [1-3]. Voici environ une quinzaine d'années, il a été proposé de l'employer afin de suivre le développement de couches formées lors de l'oxydation à haute température de matériaux métalliques [4]. Les courbes ainsi obtenues permettent de mettre en évidence l'intervention de phénomènes irréversibles, mais sans être en mesure de les identifier (leur localisation est parfois possible).

Pour aller plus avant, certains auteurs ont réalisé un enregistrement des signaux émis et proposé un classement en fonction de leur fréquence ou d'autres paramètres [5-7]. Ce classement n'est cependant pas suffisant pour identifier formellement un signal et corréler son émission à un événement précis.

Dans ce travail, nous avons choisi de réaliser un suivi par émission acoustique de l'oxydation à haute température, dans un large domaine, du zircaloy-4. Ce choix a été dicté par le fait qu'il s'agit d'une réaction présentant des périodes cinétiques bien distinctes et caractéristiques, associées à des phénomènes assez bien identifiés. Les enregistrements ont été effectués naturellement en fonction des résultats de l'étude cinétique préalable. Un premier classement a été établi grâce à une analyse de signal basée sur trois paramètres : fréquence, amplitude et énergie. Létude a été complétée par un traitement statistique des données établi sur huit paramètres. 


\section{Matériaux et techniques.}

Le zircaloy- 4 est un alliage à base de zirconium contenant essentiellement 1,5\% d'étain, $0,2 \%$ de fer et $0,1 \%$ de chrome. Loxydation a été réalisée dans l'air entre 350 et $750{ }^{\circ} \mathrm{C}$. Les prises de masse ont été suivies en continu dans une thermobalance ou en discontinu par pesées successives. Les analyses par diffraction $\mathrm{X}$ réalisées après refroidissement ou en cours d'oxydation grâce à un goniomètre équipé d'un four ont permis de déterminer la nature des phases formées.

Le suivi de l'émission acoustique a été réalisé grâce à un montage particulier déjà décrit précédemment [4]. Les signaux émis étaient enregistrés à l'aide d'un micro-ordinateur grâce auquel était, par la suite, réalisé leur traitement. Compte tenu d'impératifs particuliers (nécessité d'une cinétique suffisamment rapide pour éviter des durées d'enregistrement trop importantes mais assez lente pour que les phénomènes puissent être séparés) une seule température $\left(550^{\circ} \mathrm{C}\right)$ a été retenue pour cette partie de l'étude.

\section{Résultats cinétiques.}

L'étude cinétique que nous avons réalisée pour des durées d'oxydation allant jusqu'à quarante heures montre que les courbes prise de masse en fonction du temps présentent une allure bien particulière (Fig. 1) mais déjà bien connue [8]. Dans une première partie, la vitesse diminue progressivement durant quelques heures. Puis une accélération intervient et finalement la vitesse se stabilise avec une valeur constante. Si la température est trop faible, on observe seulement les deux premières périodes, voire même uniquement la première (c'est le cas par exemple à 370 et $400^{\circ} \mathrm{C}$ ).

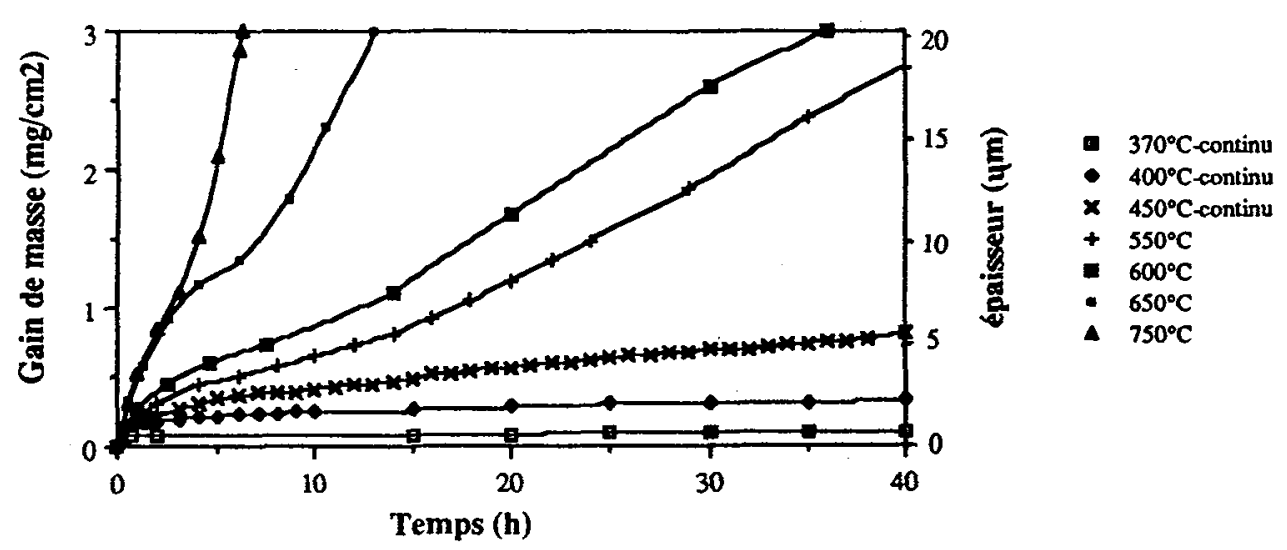

Fig. 1. - Cinétique d'oxydation du zircaloy-4 : prise de masse en fonction du temps.

L'analyse de la couche obtenue par diffraction X montre qu'il s'agit bien évidemment de zircone mais qu'elle se présente en fait sous deux formes allotropiques dont les proportions respectives varient au cours du temps : il s'agit d'une part de la forme monoclinique classique et d'autre part de la forme quadratique stable uniquement sous pression élevée. 
Sa présence indique donc l'existence de fortes contraintes en certains endroits de la couche. La proportion de phase quadratique a pu être calculée grâce aux spectres de diffraction $\mathrm{X}$ en employant la formule proposée par Garvie et Nicholson [9]. On constate que le pourcentage de zircone quadratique diminue constamment au cours de l'oxydation, rapidement au début, plus lentement par la suite (Fig. 2). Par ailleurs, les mesures réalisées en température conduisent à des teneurs en zircone quadratique plus élevées que les mesures effectuées à l'ambiante. Enfin, des essais effectués deux ans après oxydation conduisent à des teneurs encore plus faibles en phase quadratique.

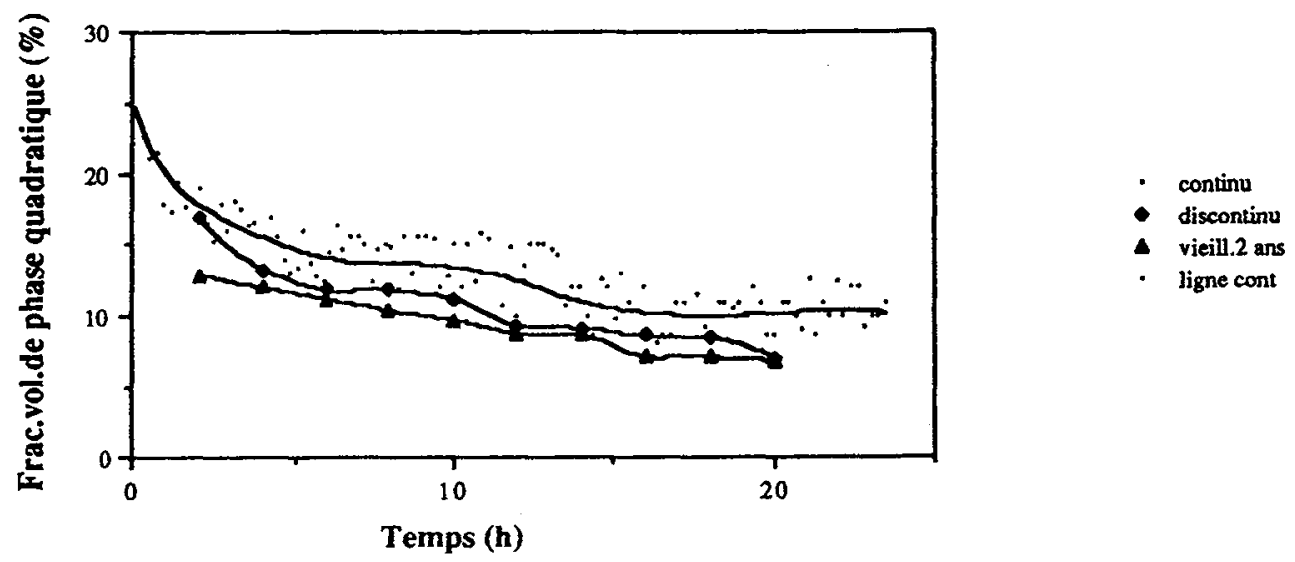

Fig. 2. - Evolution de la teneur en phase quadratique dans la couche d'oxyde : évaluation par diffraction des rayons $\mathrm{X}$.

Les observations morphologiques montrent clairement que dans la période initiale (ralentie) la couche de zircone formée est compacte, assez régulière et adhérente au métal (Fig. 3a). Par ailleurs sa couleur noire indique qu'il s'agit d'un composé non stoechiométrique. La transition cinétique est marquée par l'apparition de deux réseaux de fissures, le premier parallèle aux interfaces, le second perpendiculaire à celles-ci (Fig. 3b). Par ailleurs, la couleur de la zircone se modifie, indiquant un passage à une composition quasi stoechiométrique. Enfin des écaillages se manifestent, du moins après retour à la température ambiante.

\section{Résultats d'émission acoustique.}

Le premier essai a consisté à réaliser un simple comptage cumulé du bruit émis au cours de l'oxydation. La courbe obtenue présente une analogie certaine avec la courbe de prise de masse en fonction du temps.

Le traitement numérique du signal montre, durant la première phase de l'oxydation (partie où la vitesse diminue régulièrement) la présence de signaux de faible amplitude, peu énergétiques et riches en composantes spectrales (Type 1). Ils sont caractéristiques d'une émission acoustique dite "continue".

Au cours de la deuxième phase (accélération), on enregistre essentiellement deux familles de signaux (Type 2 et Type 3) qui ont une amplitude plus grande et une énergie moyenne 


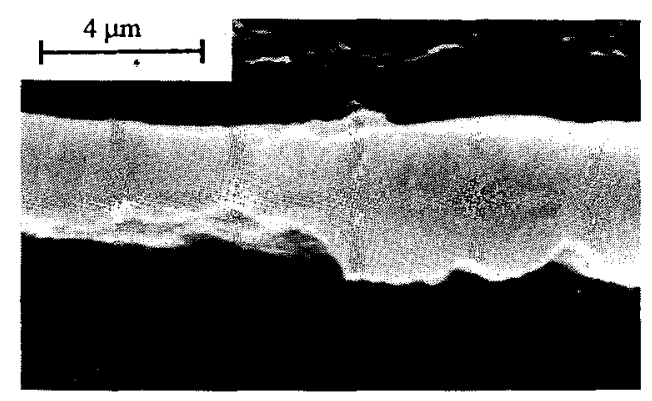

a)

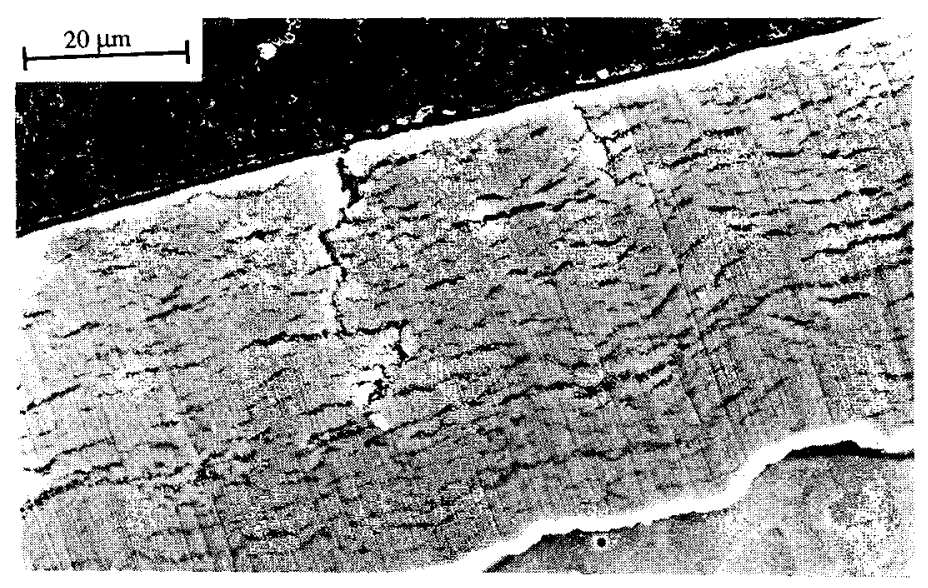

b)

Fig. 3. - Observations micrographiques en coupe de la couche d'oxyde : a) après 4 h d'oxydation à $550^{\circ} \mathrm{C} ; \mathrm{b}$ ) après $119 \mathrm{~h}$ d'oxydation à $550^{\circ} \mathrm{C}$.

localisée en une ou plusieurs composantes fréquentielles. Ils donnent lieu à une émission dite "burst" ou par salve.

Durant la troisième partie enfin (linéaire), on trouve en plus une émission constituée de la superposition de signaux (type salve + continu), qui sont très énergétiques (certains même saturant l'enregistrement).

Lanalyse de quelques paramètres des signaux (amplitude, énergie, fréquence) a permis de caractériser les différentes phases de l'oxydation et a montré une corrélation entre l'émission acoustique et la cinétique de la réaction. Malgré les résultats obtenus, cette méthode seule s'avère toutefois insuffisante pour la reconnaissance et la discrimination des différents mécanismes tout en étant très lourde à mettre en oeuvre (un millier de signaux à traiter environ).

Une autre approche peut être d'une aide efficace ; il s'agit du traitement de données par analyse en composantes principales (ACP). Cette technique intègre l'ensemble des données d'un essai ( $8 \times 1000$ dans le cas présent) pour en extraire le maximum d'informations et les représenter sous forme graphique facilement exploitable.

L'ACP s'effectue sur des tableaux de $n$ individus (signal) à $p$ variables (paramètres), elle détermine la matrice des corrélations $\left(C=X^{\prime} X\right)$ entre les variables, les axes principaux d'inertie (facteurs) et leur taux d'inertie. Le calcul de la variance de ces facteurs montre qu'ils contiennent respectivement $72,7 \%$ et $16,6 \%$ de l'inertie totale du nuage de points.

Cette méthode permet donc de réduire le nombre de dimensions sans perte significative 
d'informations ; celà consiste à chercher un sous espace qui ajuste au mieux le nuage des $n$ points dans $\mathrm{R}^{p}$. La projection des individus sur le plan formé par les axes factoriels $\mathrm{F} 1$ et F2 contiendra $89,3 \%$ de l'information ; les premiers pourcentages sont ici élevés parce qu'il existe une concentration assez nette du nuage dans un sous-espace à deux dimensions $(89,3 \%$ de variance).

L'axe factoriel est l'axe $\mathrm{F} \alpha$ qui porte le vecteur unitaire $\mathbf{u} \alpha$ (tel que $\mathbf{u}^{\prime} \cdot \mathbf{u}=1$ ). Chaque individu du tableau transformé $X$ (de terme général $x_{i j}=\left(\frac{r_{i j}-\bar{r}_{j}}{s_{j} \sqrt{n}}\right)$ ) représente un point dans l'espace $\mathrm{R}^{p}$, les $n$ lignes du vecteur $X \mathbf{u}$ sont les $n$ produits scalaires de ces points avec $\mathbf{u}$ et sont donc les coordonnées des points-individus sur l'axe $\mathrm{F} \alpha$.

Les coordonnées des points-individus sur les axes factoriels F1 et F2 sont déterminées par la combinaison suivante :

$$
\sum_{j}\left(\frac{r_{i j}-\bar{r}_{j}}{s_{j} \sqrt{n}}\right) \mathbf{u}_{i j}
$$

où $r_{i j}$ : terme général du tableau initial $; \bar{r}_{j}:$ moyenne arithmétique de la variable $j ; s_{j}:$ écart type de la variable $j ; \mathbf{u}$ : vecteur unitaire

Les pourcentages de variance associês aux axes factoriels sont de 72,2\% pour le premier, et $16,6 \%$ pour le second.

Laxe 1, largement prédominant, est corrélé positivement avec les variables les plus énergétiques. Il représente l'énergie du signal.

Laxe 2 varie dans le même sens que les variables de plus faible énergie mais situées en haute fréquence. Cet axe caractérise la composante fréquentielle du signal.

L'analyse des données de la première phase montre qu'aucun élément ne se singularise après projection dans les premiers plans factoriels [1-2] et [1-3] qui représentent $70 \%$ de l'information.

Pour la deuxième phase, la projection des individus sur le plan [1-2] fait apparaître trois classes de signaux distinctes.

Enfin pour la troisième phase de l'oxydation, la projection sur le même plan fait apparaître deux nouvelles familles de signaux par rapport aux précédentes déjà citées.

En résumé, l'ACP effectuée avec toutes les données des trois phases cinétiques a permis de montrer l'existence de cinq familles distinctes de signaux (Fig. 4).

\section{Discussion.}

Les résultats obtenus au cours de l'étude cinétique sont parfaitement semblables à ceux rapportés par Godlewski [10] lors du travail récent qu'il a réalisé concernant l'oxydation du même zircaloy-4 dans la vapeur d'eau pressurisée. La seule différence notable concerne, après la transition cinétique, l'obtention d'une zircone stoechiométrique (au moins dans la partie la plus externe) ce qui n'était pas le cas pour les réactions effectuées en autoclave. On note par contre une grande similarité en ce qui concerne la présence de la phase quadratique et l'évolution de sa concentration globale mesurée par diffraction des rayons X. Ces résultats suggèrent donc un mécanisme d'oxydation semblable à celui proposé par Godlewski : durant la première phase il existe dans la couche un gradient de contraintes, le maximum sensiblement constant se situant à l'interface interne. Ces contraintes stabilisent la zircone quadratique. Puis il arrive un moment où les contraintes à l'interface externe deviennent trop faibles. La transformation quadratique-monoclinique intervient, provoquant la formation de fissures 


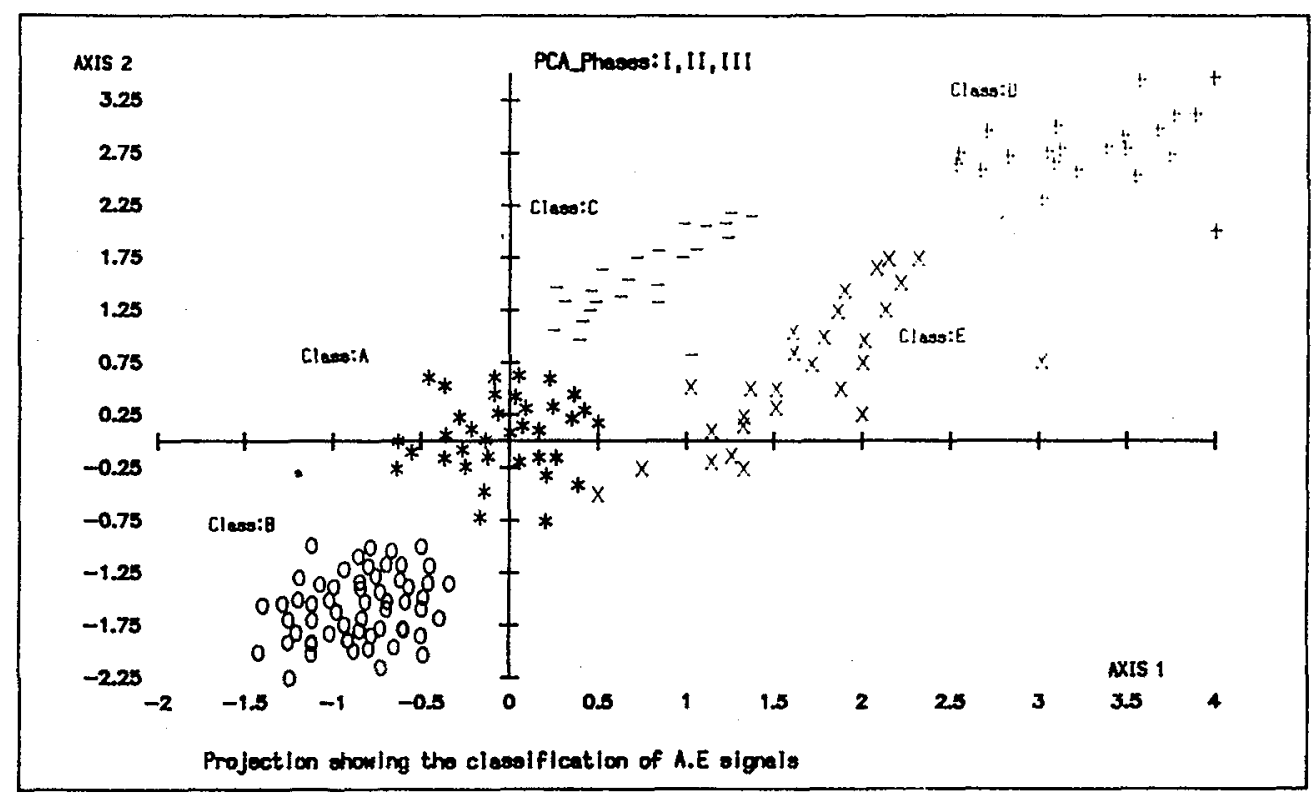

Fig. 4. - Les diverses classes de signaux déterminées par ACP.

qui elles-mêmes diminuent le niveau de contraintes dans la zone adjacente. La transformation se propage vers l'interface interne et cette période correspond à la transition cinétique (accélération). Enfin la dernière phase (vitesse constante) est atteinte lorsqu'un équilibre s'établit entre la vitesse de propagation de la zone fissurée et la vitesse d'épaississement de la couche.

Connaissant maintenant le mecanisme d'oxydation ainsi que les phénomènes et manifestations diverses qui lui sont attachés, nous pouvons tenter d'imaginer l'origine des divers signaux enregistrés. Les signaux de petite amplitude et de faible énergie formant l'émission acoustique "continue" et qui constituent la classe A mise en evidence durant la première phase peuvent être attribués à deux types d'événements : la diffusion ou la transformation quadratique-monoclinique. Il n'existe malheureusement aucun critère nous permettant de trancher entre les deux hypothèses.

Les signaux de type burst à une ou plusieurs fréquences sont générés par des processus plus énergétiques qui commencent à se manifester au deuxième stade de l'oxydation. L'ACP montre qu'en fait deux nouvelles classes se manifestent (B et $\mathrm{C}$ ), la classe A subsistant. On peut raisonnablement attribuer ces nouveaux signaux à la fissuration (création de deux types de fissures).

Enfin deux nouvelles classes de signaux (D et E) se manifestent au cours de la troisième phase de l'oxydation, sans que nous soyons en mesure toutefois de leur attribuer une origine (peut être sont-ils liés à des décollements des couches à haute température).

\section{Conclusions.}

Ce travail nous a tout d'abord permis de montrer que l'oxydation du zircaloy-4 à l'air se déroule de manière semblable à celle décrite dans la vapeur d'eau pressurisée et met donc en 
jeu probablement des phénomènes identiques. Par ailleurs l'étude par émission acoustique, complétée par un traitement des signaux et une analyse des données, montre que les signaux émis se répartissent en plusieurs classes. Certains d'entre eux peuvent être corrélés à des événements tels que la transformation de phase ou la fissuration. Pour d'autres, l'origine nous demeure encore inconnue. On constate toutefois que l'analyse mathématique adoptée se révèle un outil efficace dans la discrimination des signaux.

\section{Bibliographie}

[1] MARTIN P., DICKSON J.I., BAILON J.P., Mater. Sci. Eng. 69 (1985) 9.

[2] HAY D.R., Chan R.W.Y., SHARP D., SIdDIQUi K.J., J. Acoust. Emission 3 (1984) 118.

[3] GRAHAM L.J., ElSLEY R.K., J. Acoust. Emission 2 (1983) 47.

[4] Coddet G., Beranger G., Ghretien J.F., Materials and Coatings to Resist High Temperature Corrosion, D.R. Holmes, A. Rahmel Eds. (Applied Science Publishers, London, 1978) p. 175.

[5] BennetT M.J., Buttle D.J., Colledge P.D., Price J.B., Scruby C.B., STacey K.A., Mater. Sci. Eng. 120 (1989) 199.

[6] KHanna A.S., JHA B.B., Baldev R., Oxid. Met. 27 (1987) 95.

[7] Baldev R., JHa B.B., Khanna A.S., Battacharya D.K., Int. J. Pres. Ves. Piping 45 (1991) 301.

[8] ANTONi-le Guyadec F., Thèse de Doctorat, Institut National Polytechnique de Grenoble (1990).

[9] Garvie R.C., Nicholson P.S., J. Am. Ceram. Soc. 55 (1972) 303.

[10] Godlewski J., Thèse de Doctorat, Université de Technologie de Compiègne (1990). 